\title{
GENERALIZED SPECTRUM AND A PROBLEM OF APOSTOL
}

\author{
MOSTAFA MBEKHTA
}

(Communicated by Palle E. T. Jorgensen)

\begin{abstract}
We give a complete answer to a question of Apostol concerning the asymptotic behaviour of the reduced minimum modulus of a bounded operator on Hilbert space.
\end{abstract}

Let $H$ be a Hilbert space and $B(H)$ the algebra of bounded operators of $H$. For $A \in B(H)$, we denote by $N(A)$ and $R(A)$, respectively, the kernel and the range of $A$.

Define the reduced minimum modulus $\gamma(A)$ of $A$ by setting

$$
\gamma(A)=\inf \left\{\|A u\| ; u \in N(A)^{\perp} \text { and }\|u\|=1\right\}
$$

if $A \neq 0$; in this paper it will be convenient to take $\gamma(A)=0$ if $A=0$ (see Apostol [1]). It is well known (see [2, Chapter IV, §5]) that $\gamma(A)=\gamma\left(A^{*}\right)$ and that $\gamma(A)>0 \Leftrightarrow R(A)$ is closed.

Following Apostol, put

$$
\sigma_{\gamma}(A)=\left\{\mu \in \mathbf{C} ; \lim _{\lambda \rightarrow \mu} \gamma(A-\lambda I)=0\right\}
$$

and

$$
\rho_{\gamma}(A)=\mathbf{C} \backslash \sigma_{\gamma}(A) .
$$

In $[3,4]$ we give a generalization of the spectrum in which invertibility is replaced by the existence of an "analytic generalized resolvent": we put

$$
\operatorname{reg}(A)=\left\{\lambda \in \mathbf{C}: \forall n \geq 1, N(A-\lambda I)^{n} \subseteq R(A-\lambda I) \text { closed }\right\} .
$$

It is familiar that $N(A-\lambda I)^{n} \subseteq R(A-\lambda I) \Leftrightarrow N(A-\lambda I) \subseteq R(A-\lambda I)^{n}, \operatorname{reg}(A)$ is open, and $\rho(A) \subseteq \operatorname{reg}(A)$. When $0 \in \operatorname{reg}(A)$ we call $A$ "regular." The generalized spectrum of $A$, denoted by $\sigma_{\mathrm{g}}(A)$, is the complement of $\operatorname{reg}(A)$ in $\mathrm{C}$ and has properties analogous to the classical spectrum $\sigma(A)$ of $A$ (see [4]). Also [4, Corollaire 2.4] shows that $\sigma_{\mathrm{g}}(A)=\sigma_{\gamma}(A)$.

Theorem 1. Let $A \in B(H)$. Then the following conditions are equivalent:

(1) $A=A^{\prime} \oplus Q$, where $Q$ is nilpotent and $0 \in \operatorname{reg}\left(A^{\prime}\right)$.

(2) 0 is an isolated point of $\sigma_{\mathrm{g}}(A)$ and $\lim _{n \rightarrow \infty} \gamma\left(A^{n}\right)^{1 / n}$ exists and is strictly positive.

Received by the editors June 24, 1991 and, in revised form, November 5, 1991.

1980 Mathematics Subject Classification (1985 Revision). Primary 47A53, 47A55, 47B05.

Key words and phrases. Generalized spectrum, reduced minimum modulus. 
Proof. (1) $\Rightarrow$ (2) If $A=A^{\prime} \oplus Q$ (topological direct sum, not necessarily orthogonal), then there exist closed subspaces $M, N \subset H$, which are $A$-invariant such that $H=M \oplus N, A^{\prime}=A_{\mid M}$, and $Q=A_{\mid N}$. Since the operator $Q$ is nilpotent, there is $d \in \mathbf{N}^{*}$ such that $Q^{d}=0$. Therefore we have $N \subset N\left(A^{d}\right)$. To begin we show that for all $\lambda \neq 0, N(A-\lambda I) \subset M$. Let $u \in N(A-\lambda I)$. Then $u=v+w$, where $v \in M$ and $w \in N$. Furthermore,

$$
0=(A-\lambda I) u=(A-\lambda I) v+(A-\lambda I) w
$$

implies

$$
(A-\lambda I) v=-(A-\lambda I) w \in M \cap N=\{0\} .
$$

So we have $(A-\lambda I) w=0$ and $A w=\lambda w$. Hence $\forall n \geq 0, A^{n} w=\lambda^{n} w$.

So $w \in N \subseteq N\left(A^{d}\right) \Rightarrow 0=A^{d} w=\lambda^{d} w$. Since $\lambda \neq 0$, it follows that $w=0$ and therefore $u=v \in M$. Hence $N(A-\lambda I) \subseteq M$ and

$$
N(A-\lambda I)=N\left((A-\lambda I)_{\mid M}\right) .
$$

Since the operator $A_{\mid M}$ is regular, there is a neighbourhood $\mathscr{U}$ of $0 \in \mathbf{C}$ such that $\forall \lambda \in \mathscr{U},(A-\lambda I)_{\mid M}$ is regular (since $\operatorname{reg}\left(A_{\mid M}\right)$ is open). So $R\left((A-\lambda I)_{\mid M}\right)$ is closed $\forall \lambda \in \mathscr{U}$. Furthermore, $\forall \lambda \neq 0, N(A-\lambda I)=N\left((A-\lambda I)_{\mid M}\right)$. This implies that for all $\lambda \in \mathscr{U}-\{0\}$, and for all $n \geq 0$, one has $N\left((A-\lambda I)^{n}\right)$ $=N\left(\left((A-\lambda I)_{\mid M}\right)^{n}\right) \subseteq R\left((A-\lambda I)_{\mid M}\right)$. Therefore, $\forall \lambda \in \mathscr{U}-\{0\}, \forall n \geq 0$, $N\left((A-\lambda I)^{n}\right) \subset R(A-\lambda I)$.

Now we prove that $R(A-\lambda I)$ is closed for all $\lambda \in \mathscr{U}-\{0\}$. The operator $A_{\mid N}$ being nilpotent, for all $\lambda \neq 0,(A-\lambda I)_{\mid N}$ is invertible in $B(N)$, hence $N=R\left((A-\lambda I)_{\mid N}\right)$. Consequently, for all $\lambda \in \mathscr{U}-\{0\}$, we have that $R(A-\lambda I)=$ $R\left((A-\lambda I)_{\mid M}\right) \oplus N$. This implies that $R(A-\lambda I)$, as a sum of two closed subspaces of $M$ and $N$, is closed. Hence $\mathscr{U}-\{0\} \subseteq \operatorname{reg}(A)$, where such 0 is isolated in $\sigma_{\mathrm{g}}(A)$.

Furthermore, [4, Théorème 3.1, Corollaire 3.9] show that $\lim _{n \rightarrow \infty} \gamma\left(A^{n}\right)^{1 / n}$ exists and is strictly positive.

(2) $\Rightarrow$ (1) follows [1, Proposition 3.3].

Remark. The implication (1) $\Rightarrow(2)$ of Theorem 1 implies that in [1, Proposition 3.3], $\sigma=\{0\}$. Furthermore, [1, Theorem 3.5] implies also that $\sigma=\{0\}$.

Proposition 2. Let $A \in B(H)$ such that $0 \in \partial \sigma(A)$, the boundary of $\sigma(A)$. Then $\overline{\lim }_{n \rightarrow \infty} \gamma\left(A^{n}\right)^{1 / n}>0$ implies that 0 is isolated in $\sigma(A)$.

Proof. We remark that $\partial \sigma(A) \subseteq \sigma_{\mathrm{g}}(A)$ (see [4, Corollary 2.2]). Using [1, Theorem 3.5], we see that 0 is isolated in $\sigma_{\mathrm{g}}(A)$. Hence there is a neighbourhood $\mathscr{U}$ of $0 \in \mathbf{C}$ such that $\mathscr{U}-\{0\} \subseteq \operatorname{reg}(A)$. Since $0 \in \partial \sigma(A)$, it follows that $(\mathscr{U}-\{0\}) \cap \rho(A) \neq \varnothing$. Consequently, $\mathscr{U}-\{0\} \subseteq \rho(A)$ (see [4, Corollary 2.3]). The proposition is proved.

Corollary 3. If $0 \in \partial \sigma(A)$, then

$$
0 \text { not isolated in } \sigma(A) \Rightarrow \varlimsup_{n \rightarrow \infty} \gamma\left(A^{n}\right)^{1 / n}=0 \text {. }
$$

Proof. Direct consequence of Proposition 2.

Remark. (1) If 0 is isolated in $\sigma(A)$ then both cases are possible.

For example, if $A$ is selfadjoint and not invertible, with closed range $R(A)$, then $H=N(A) \oplus R(A)$ and for all $n>0$, we have $\gamma\left(A^{n}\right)=\gamma(A)^{n}$. It is clear that 0 is isolated in $\sigma(A)$, and since $R(A)$ is closed, we have $\gamma(A)>0$. 
If instead $A$ is nilpotent then $\lim _{n \rightarrow \infty} \gamma\left(A^{n}\right)^{1 / n}=0$ since $\gamma(A)=0$ if $A=0$. (2) Corollary 3 and the preceding examples give a complete answer to Question 1 posed by Apostol [1, p. 289].

\section{REFERENCES}

1. C. Apostol, The reduced minimum modulus, Michigan Math. J. 32 (1985), 279-294.

2. T. Kato, Perturbation theory for linear operators, Springer, New York, 1966.

3. M. Mbekhta, Généralisation de la décomposition de Kato aux opérateurs paranormaux et spectraux, Glasgow Math. J. 29 (1987), 159-175.

4. __ Résolvant généralisé et théorie spectrale, J. Operator Theory 21 (1989), 69-105.

U.F.R. De MathématiQues, Universiteé de Lille I, 59655-Villeneuve D’AscQ, France 